\title{
A case of Henoch-Schönlein purpura associated with scrub typhus
}

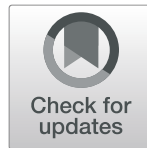

\author{
Jae Hyoung Im', Suk Jin Choi ${ }^{2}$, Moon-Hyun Chung ${ }^{3}$, Seung Yun Lee ${ }^{4}$, Young Kyoung Park', Hea Yoon Kwon', \\ Ji Hyeon Baek ${ }^{1}$ and Jin-Soo Lee ${ }^{1 *}$
}

\begin{abstract}
Background: Henoch-Schönlein purpura (HSP) may be caused by several allergens. However, to date, HSP caused by Orientia tsutsugamushi has not been reported. Here, we report an unusual rash with features of HSP caused by Orientia tsutsugamushi.

Case presentation: A man visited a tertiary hospital with bilateral symmetrical purpura and fever. He presented with an eschar in the left popliteal fossa and proteinuria. He was diagnosed with tsutsugamushi disease by indirect fluorescent antibody and positive polymerase chain reaction tests. Purpura biopsy demonstrated a feature of leukocytoclastic vasculitis and IgA deposition in dermal vessels, indicative of HSP.

Conclusions: When examining patients with unique rashes, such as in this case, we suggest investigating out-door activities and evidence of mite bites. Furthermore, differential diagnosis of tsutsugamushi disease should be considered when necessary.
\end{abstract}

Keywords: Cutaneous, Leukocytoclastic, Orientia tsutsugamushi, Henoch-Schönlein, Scrub typhus, Case report

\section{Background}

Scrub typhus is a febrile disease caused by Orientia tsutsugamushi (O. tsutsugamushi), and is transmitted by infected Leptotrombidium mites [1]. It is characterized by fever, lymphadenopathy, rash, and typical eschar. It causes various complications such as bronchitis, pneumonia, myocarditis, and meningitis [2]. Endothelial activation by Orientia tsutsugamushi induces the immune response by promoting the production of interleukins and tumor necrosis factor, which can manifest as various symptoms [3]. Henoch-Schönlein purpura (HSP) is a relatively common vasculitis associated with or induced by infection [4]; however, HSP due to scrub typhus has not been reported. Here, we describe a patient who

\footnotetext{
*Correspondence: ljinsoo@inha.ac.kr; ljinsoo16@gmail.com

'Division of Infectious Diseases, Department of Internal Medicine, Inha University College of Medicine, 7-206, Shinheung-Dong, Jung-Gu, Incheon 22332, Republic of Korea

Full list of author information is available at the end of the article
}

presented with HSP and an atypical rash, potentially triggered by scrub typhus.

\section{Case presentation}

A 52-year-old man was admitted to a tertiary hospital (Incheon, Republic of Korea) due to a febrile rash in November. He had diabetes mellitus and hypertension without any complications. At the time of his visit, his blood pressure was $140 / 90 \mathrm{mmHg}$, body temperature was $39.9^{\circ} \mathrm{C}$, and heart rate was 107 beats/minute. He did not exhibit any respiratory or gastro-intestinal symptoms or arthralgia. He presented with eschar in the left popliteal fossa. He had a maculopapular rash on his trunk, and palpable purpura was remarkable in the lower extremities (Fig. 1). His leukocyte count was 11,840 cells/ $\mu \mathrm{L}$, hemoglobin was $14.5 \mathrm{~g} / \mathrm{dL}$, and platelets were 274 , 000 cells $/ \mu \mathrm{L}$. The aspartate transaminase/alanine transaminase ratio was slightly elevated to $55 / 85 \mathrm{IU} / \mathrm{L}$ and total bilirubin level $(0.8 \mathrm{mg} / \mathrm{dL})$ was normal. The patient had elevated erythrocyte sedimentation rate $(22 \mathrm{~mm} /$

(c) The Author(s). 2020 Open Access This article is licensed under a Creative Commons Attribution 4.0 International License, which permits use, sharing, adaptation, distribution and reproduction in any medium or format, as long as you give appropriate credit to the original author(s) and the source, provide a link to the Creative Commons licence, and indicate if changes were made. The images or other third party material in this article are included in the article's Creative Commons licence, unless indicated otherwise in a credit line to the material. If material is not included in the article's Creative Commons licence and your intended use is not permitted by statutory regulation or exceeds the permitted use, you will need to obtain permission directly from the copyright holder. To view a copy of this licence, visit http://creativecommons.org/licenses/by/4.0/. The Creative Commons Public Domain Dedication waiver (http://creativecommons.org/publicdomain/zero/1.0/) applies to the data made available in this article, unless otherwise stated in a credit line to the data. 

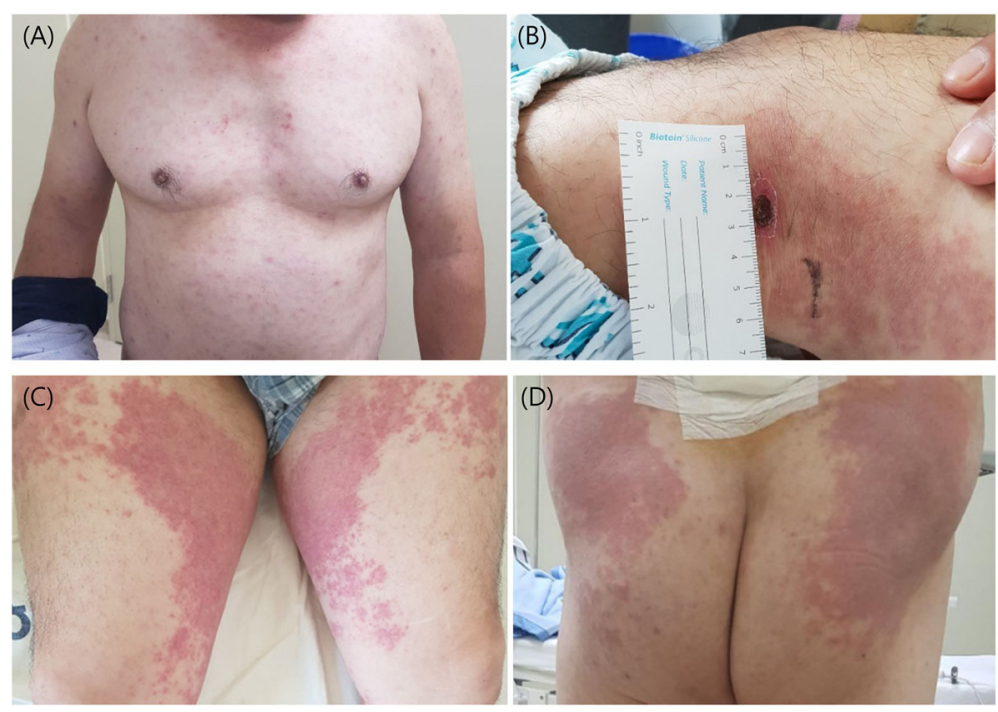

Fig. 1 Images show (a) maculopapular rash on the trunk, (b) eschar on the left popliteal fossa, (c) palpable purpura on both lower limbs, and (d) palpable purpura on both buttocks

hour) and C-reactive protein $(9.57 \mathrm{mg} / \mathrm{dL})$ values. The urine dipstick test showed proteinuria $(++)$ without pyuria or bacteriuria, and autoantibodies were negative. Although IgM (indirect fluorescent antibody, 1:64), IgG titer (1: 256), and western blot were positive for Lyme disease (Borrelia serology, tested by Korea Center for Diseases Control and prevention [5]), the patient did not present erythema migrans or arthralgia, which are typical findings of Lyme disease. Six weeks later, Borrelia IgM was negative and IgG titer decreased to 1:64, in the
Lyme disease test. O. tsutsugamushi IgM and IgG titers (indirect fluorescent antibody, Supplementary 1) were 1: 1024 and 1:2048, respectively. Further, O. tsutsugamushi IgA titer was also high (1:1024). The $56 \mathrm{kDa}$ antigen gene of $O$. tsutsugamushi was positive by polymerase chain reaction on the first day at the hospital (Supplementary 1). Histological examination of the skin biopsy of the rash showed findings of leukocytoclastic vasculitis and deposition of IgA on dermal blood vessel walls (Fig. 2). Rash, histologic findings, and proteinuria

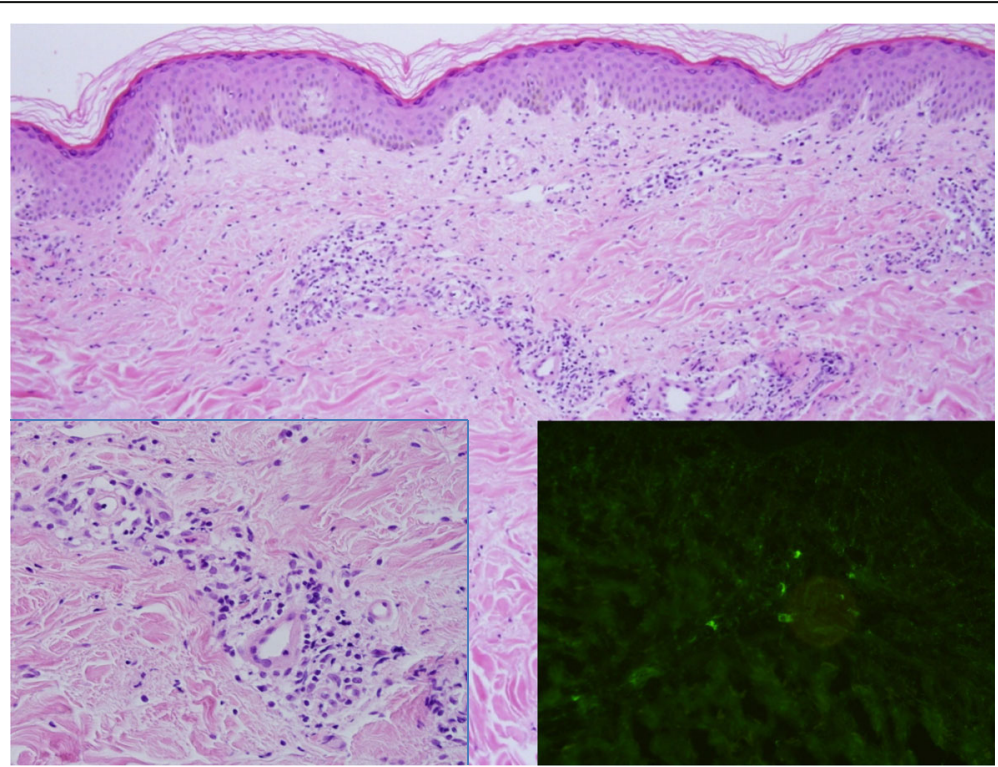

Fig. 2 The biopsy of the skin lesion showed perivascular infiltration of inflammatory cells in the upper dermis along with conspicuous karyorrhectic debris (original magnification $\times 100$; left lower Insert, $\times 400$ ) and the presence of IgA deposition on the wall of the blood vessels (direct immunofluorescence test, right lower insert, $\times 200)$ 
satisfied the criteria for diagnosis of Henoch-Schönlein purpura. He had not started any new medications for a month and had no symptoms of any infections other than scrub typhus. Thus, the patient was diagnosed with Henoch-Schönlein purpura associated with scrub typhus. Despite no immune suppressive treatment, the patient showed a dramatic response to doxycycline (100 mg twice/day) alone. His fever resolved within $24 \mathrm{~h}$, and the rash (both maculopapular rash on his trunk and palpable purpura on his lower extremities) began to improve within $48 \mathrm{~h}$. He was discharged after 4 days. Five weeks later, his rash and proteinuria improved, and he is currently living without any sequelae.

\section{Discussion and conclusions}

The case described herein presented without the typical arthritis or abdominal pain of the classic triad of HSP. However, the 2010 European League Against Rheumatism criteria were satisfied [6]. There have been several cases of HSP associated with Rickettsia spp. [7] and Bartonella spp. [8], which are believed to share a common ancestor with $O$. tsutsugamushi. However, scrub typhusinduced HSP has not been reported to date.

Although the pathogenesis of HSP is unclear, it is well known that 30 to $50 \%$ of patients are predisposed to upper respiratory tract infection. Streptococcus, mycoplasma, hepatitis B virus, herpesvirus, parvovirus, coxsackievirus, adenovirus, measles, and rubella are known to cause HSP [9]., In addition to infection, other allergens such as vaccination, drugs, food, and insect bites are also considered as causative agents of HSP [10]. The main pathophysiology of HSP is the presence of abnormal immunoglobulin A (IgA) deposits on the vessel wall [11]. Our patient presented with elevated serum O. tsutsugamushi IgA antibodies (1:1024) and there was evidence of IgA deposition based on histopathology evaluation. This suggested that the elevated IgA was due to $O$. tsutsugamushi caused by vasculitis. Aberrant glycosylation of IgA1 is thought to cause HSP [12], and various bacterial and viral pathogens are known to produce sialidase (neuraminidase) [13]. Decreases in sialic acid and Gal may affect IgA1 molecules, leading to the immunological mechanisms of HSP [14]. IgA1 molecules lacking sialic acid or Gal tend to aggregate to form macromolecular complexes, which activate some cytokines and the complement system, and adversely affect the endothelium [14]. In addition, sialic acid-deficient IgA tends to be preferably deposited in the kidneys [11].

In this case, the seropositivity of the Lyme disease test can lead to confusion in the interpretation of the patient's clinical features. There are three possibilities for seropositivity in Lyme disease: i) co-infection, ii) false positivity in the Lyme disease test, or iii) recrudescence of Lyme disease. Of these, the possibility of co-infection and recrudescence was very low in our case, considering the low incidence of Lyme disease in South Korea [15] and the absence of erythema migrans and arthralgia. The decrease in IgG titer from 1: 256 to 1:64 in 6 weeks also suggests no acute Lyme disease. A previous report showed that extremely high serum IgM levels induced by other pathogens could produce false positive results in the serology testing for Lyme disease (including western blot) [16]. In this case, there was a very high titer of IgM, which could have resulted from the seropositivity for scrub typhus.

This case was accompanied by eschar, which is a typical finding of O. tsutsugamushi. Antibiotics were administered promptly, even before the pathogen could be confirmed. However, depending on the patient's skin color and immunity, no or small-sized eschar may often be encountered in clinical practice [17]. Thus, it is important to identify the potential exposure to vectors and outdoor activities of patients presenting with HSP. Early use of anti-microbial agents such as doxycycline, chlorampheni$\mathrm{col}$, and azithromycin can be imperative in preventing the severity and complications related to scrub typhus.

In conclusion, we report a patient presenting with HSP, potentially triggered by $O$. tsutsugamushi. To facilitate early intervention and prevent complications it is important to consider the possibility of infections such as scrub typhus in the differential diagnosis of unusual rashes.

\section{Supplementary information}

Supplementary information accompanies this paper at https://doi.org/10 1186/s12879-020-05001-X.

\section{Additional file 1.}

Abbreviations

HSP: Henoch-Schönlein purpura; Ig: Immunoglobulin

Acknowledgements

Not applicable.

Authors' contributions

JHI: drafting and revision of the manuscript. SJC: histopathology analysis.

YKP: interpretation of laboratory tests. MHC, SYL, HYK, JSL, and JHB:

discussion and revision of the manuscript. JSL: final approval of the manuscript version for publication. All authors read and approved the final manuscript.

\section{Funding}

This work was supported by the National Research Foundation of Korea (NRF) grant funded by Korea's government (MSIT) (No. 2017R1A6A3A11028403 and 2017R1D1A1B03029939).

Availability of data and materials

The datasets used during the current study are available from the corresponding author upon reasonable request.

Ethics approval and consent to participate Not applicable.

Consent for publication

Written informed consent (including for photographs that may identify the patient) was obtained from the patient for publication of this case report. 


\section{Competing interests}

The authors declare that they have no competing interests.

\section{Author details}

'Division of Infectious Diseases, Department of Internal Medicine, Inha University College of Medicine, 7-206, Shinheung-Dong, Jung-Gu, Incheon 22332, Republic of Korea. ${ }^{2}$ Department of Pathology, Inha University College of Medicine, Incheon, Republic of Korea. ${ }^{3}$ Department of Internal Medicine, Seogwipo Medical Center, Jeju, Jeju-do, Republic of Korea. ${ }^{4}$ Division of Rheumatology,Department of Internal Medicine, Inha University College of Medicine, Incheon, Republic of Korea. ${ }^{5}$ Translation Research Center, Inha University College of Medicine, Incheon, Republic of Korea.

Received: 12 October 2019 Accepted: 29 March 2020

Published online: 17 April 2020

\section{References}

1. Bennett JE, Dolin R, Blaser MJ. Principles and practice of infectious diseases, vol. 1: Elsevier Health Sciences; 2014.

2. Luce-Fedrow A, Lehman M, Kelly D, Mullins K, Maina A, Stewart R, Ge H, John H, Jiang J, Richards AJTm et al: A review of scrub typhus (Orientia tsutsugamushi and related organisms): then, now, and tomorrow. 2018, 3(1):8.

3. Otterdal K, Janardhanan J, Astrup E, Ueland T, Prakash JA, Lekva T, Abraham $\mathrm{O}$, Thomas K, Damås JK, Mathews P. Increased endothelial and macrophage markers are associated with disease severity and mortality in scrub typhus. J Infect. 2014;69(5):462-9.

4. Saulsbury F. Epidemiology of Henoch-Schonlein purpura. Cleve Clin J Med. 2002;69:SII-87.

5. Park S-H, Hwang K-J, Chu H, Park M-Y. Serological detection of Lyme borreliosis agents in patients from Korea, 2005-2009. Osong Public Health Res Perspect. 2011;2(1):29-33.

6. Ozen S, Pistorio A, lusan SM, Bakkaloglu A, Herlin T, Brik R, Buoncompagni A, Lazar C, Bilge I, YJAotrd U. EULAR/PRINTO/PRES criteria for HenochSchönlein purpura, childhood polyarteritis nodosa, childhood Wegener granulomatosis and childhood Takayasu arteritis: Ankara 2008. Part II: Final classification criteria. 2010;69(5):798-806.

7. Nigwekar P, Kavar Y, Shrikhande D. Unusual Presentation of Rickettsial Fever as Henoch-Schonlein Purpura: A Case Report. Pravara Med Rev. 2012:4(3).

8. Ayoub EM, Mcbride J, Schmiederer M, Anderson BJTPidj: Role of Bartonella henselae in the etiology of Henoch-Schönlein purpura 2002, 21(1):28-31.

9. Rai A, Nast C, Adler S. Henoch-Schonlein purpura nephritis. J Am Soc Nephrol. 1999;10(12):2637-44.

10. Szer IS. Henoch-Schonlein purpura. Curr Opin Rheumatol. 1994;6(1):25-31.

11. Lau KK, Suzuki H, Novak J, Wyatt RJ. Pathogenesis of Henoch-Schönlein purpura nephritis. Pediatr Nephrol. 2010;25(1):19.

12. Saulsbury F. Alterations in the O-linked glycosylation of $\operatorname{lgA} 1$ in children with Henoch-Schönlein purpura. J Rheumatol. 1997;24(11):2246-9.

13. Moncla B, Braham P, Hillier S. Sialidase (neuraminidase) activity among gram-negative anaerobic and capnophilic bacteria. J Clin Microbiol. 1990; 28(3):422-5

14. Novak J, Julian BA, Tomana M, Mestecky J. IgA glycosylation and IgA immune complexes in the pathogenesis of IgA nephropathy. In: Seminars in nephrology: 2008: Elsevier; 2008. p. 78-87.

15. Im JH, Baek J, Durey A, Kwon HY, Chung M-H, Lee J-SJV-B, Diseases Z: Current status of tick-borne diseases in South Korea. 2018.

16. Gregson D, Evans G, Patrick D, Bowie W. Lyme disease: How reliable are serologic results? CMAJ. 2015;187(16):1193-4.

17. Kim YH, Hyun W, Kim DP, Chung M-H, Im JH, J-h B, Lee J-S, Kang J-S. The Eschar size and early inoculation lesion of Tsutsugamushi disease on Jeju Island, Korea. Infect Chemother. 2019;51(4):345-54.

\section{Publisher's Note}

Springer Nature remains neutral with regard to jurisdictional claims in published maps and institutional affiliations. 